\title{
Trends towards Multi-Radio and Multi-Channel based Wireless Mesh Networks
}

\author{
Damanjit Kaur \\ M.Tech Student \\ Department of Computer Science and Engineering \\ Guru Nanak Dev University, Amritsar
}

\begin{abstract}
This paper is a review on QoS issue of wireless mesh networks. QoS comes up with a great collection of networking technologies and procedures that guarantees the capability of a network to deliver with predictable consequences. WMNs have emerged as a flexible, reliable and cost effective way of providing broadband internet access over wide areas through multi hop communication. This paper has reviewed different routing protocols used in the WMNs. The overall objective of this paper is to explore the various short comings of the routing protocols of WMNs.
\end{abstract}

\section{General Terms}

WMNs, QoS, flooding, collision avoidance, secure transmission, congestion control.

\section{INTRODUCTION}

Wireless Mesh Networks (WMNs) is just a key technology, supporting a variety of several emerging and commercially interesting applications e.g., neighborhood networks, community and broadband home networking. To offer adaptive and flexible wireless Internet connectivity, it has been evolved as you of the very promising concepts for autoconfigurable and self-organizing wireless network with multi hop and mesh technology. Network connectivity is automatically maintained and established by most of the nodes in the mesh network. A communication network comprised of radio nodes which are structured in a mesh topology is recognized as wireless mesh networks. It contains mesh clients, mesh routers and gateways. The mesh client include laptops, mobile phones and wireless devices and mesh routers forward traffic to and from the gateways required for connecting to internet. The reliability and redundancy are properties with this network. WMN can be implemented with a range of wireless technology offering 802.11, 802.16, cellular technologies or combining several type [1].

Additionally it serves as a cost effective technique for establishing robust and reliable broadband access between Online sites providers (ISPs) and other end-users. The typical architecture of WMNs contains three distinct elements: mesh gateway, mesh routers and mesh clients. Static mesh routers form the wireless backbone; mesh clients interconnect to each other and access the network through mesh routers. The next are technical requirements wanted by mesh networks

The high capacity necessities of the access nodes that forward the accumulated traffic of the essential users should be met by WMN.

- The multiple strict quality-of-service (QoS) requirements of the end user applications, including end-to-end (ETE) packet delay, throughput, and packeterror-rate (PER) should be managed.

- The most truly effective communication range to guarantee that no APs (or groups of APs) are isolated from the Internet gateways need to be provided. [2]

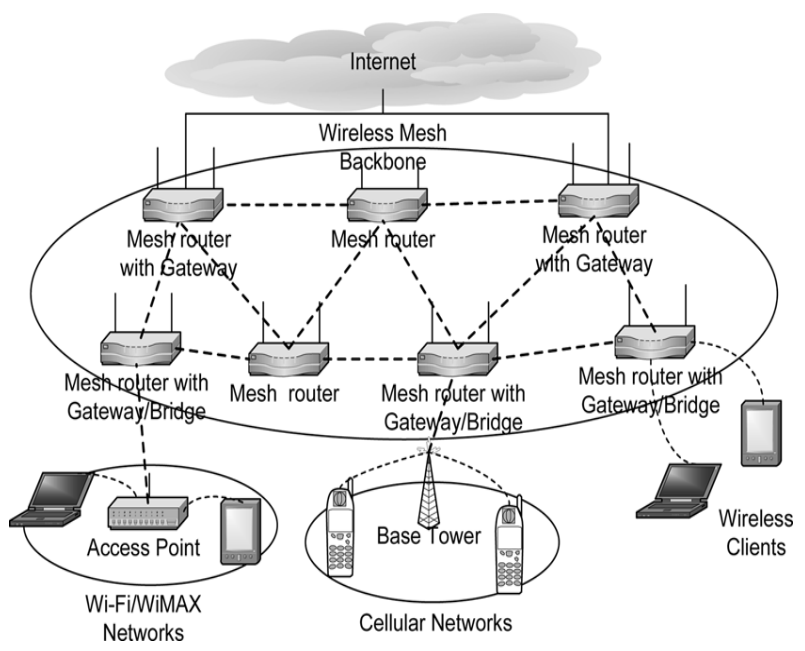

Figure 1: Network Architecture of wireless mesh network

The multi-hop nature of WMNs and the rapid growth of throughput demands lead to multi- channels and multi-radios structures in mesh networks, nevertheless the interference of co-channels, as a problem reduces the sum total throughput, especially in multi-hop networks. Therefore, the utilization of orthogonal channels and multiple-radios are two efficient solutions for decreasing co-channels interference and improving the network throughput.

\subsection{QoS in Wireless Mesh Networks}

QoS (Quality of Service) describes a vast number of networking technologies and techniques that guarantees the power of a network to supply with predictable results. Various elements of network performance that can come under the scope of QoS often include availability (uptime), bandwidth (throughput), latency (delay), and error rate. QoS involves prioritization of network traffic. QoS could be inclined to a network interface, towards a particular server or router's performance, or in specific applications. So as to insure that networks are performing at the required level, a network monitoring system could be deployed as part of QoS.

For supporting audio and video, real time communications, QoS provisioning is quite definitely essential. As set alongside the wired networks, the provisioning for QoS of wireless networks is far tougher concept as a result of following reason:

-The unavailability of any central coordination authority 
- Mobility of node

- Limited electric batteries,

- Multi-hop communication

-Disputation for accessing the wireless channel

\subsection{QoS Routing}

Due to interference among various transmissions, the QoS routing in multi-hop wireless networks is formidable task. In case there is multi-channel wireless network, since two transmissions utilizing the same channel may enter together so there exists interference. The request of QoS connection usually accompanies bandwidth requirement and QoS routing seeks a source to destination route with requested bandwidth [16].

The wireless mesh routing protocol offers QoS guarantees from source to destination when its interacting end-points fall inside the network range and from the client to the net gateway when the traffic is directed to the internet. The QoS metrics are maximum end-to-end delay and minimum bandwidth. The available stable route is selected by the protocol when multiple routes are present.

The many benefits of WMNs are the following:

- Moderate up-front cost,

- Facile network maintenance

- Robustness

- Reliable service coverage

- Rapidly deployable, self-healing, self-organizing and it have low transmission power.

The key objectives of QoS based routing are optimal utilization of resources for improving total network throughput and graceful performance degradation during overloading conditions offering better throughput, Dynamic determination of feasible paths for accommodating the QoS of the given flow under various policy constraints such as provider selection, path cost etc. [4]

\section{Routing Protocols}

There are various criteria for classifying and designing routing protocols for wireless networks. For example what, when and the way the routing information is exchanged, when and the way the routes are computed. [6][4]

There exist various routing protocols that may be grouped as: periodic or proactive, on demand or reactive and hybrid protocols. Proactive protocols does what exactly beforehand, it already determines the routes to various nodes so that when required they may be used. It requires to be in a continuously updated state of the network. As all of the routes are to be discovered, the route discovery overheads increases with frequent topology changes and the it is difficult to steadfastly keep up and update the info in the tables.

This may cause delay in data packets or even packets could be lost leading to the reduced performance of the network. Various proactive protocols are Destination-Sequenced Distance Vector Routing Protocol (DSDV) AND Wireless Routing Protocol (WRP) [1]. Reactive protocols do not determine the routes beforehand but rather create and maintain routes only on demand .Hence they're in general more scalable in nature [7].
Examples of such protocols are DSR and AODV. Hybrid protocol is a basic routing protocol that is dependant on AODV and tree-based routing. It supports two kinds of path selection protocols. It uses peer link management protocol that uses mesh point to find out and track neighbouring nodes [17].

Destination Sequenced Distance Vector Routing Protocol (DSDV): DSDV, produced by Perkins and Royer (1999) is a table driven routing centered on Bellman-Ford algorithm. The main purpose to produce it was to fix routing loop problem. The routing table maintains each entry using the sequence number, which if link is present is even else odd. This sequence number is generated by the destination and emitter needs to send this number with another update. Routing information is distributed one of the nodes by frequently sending small incremental updates and sending infrequently full dumps (Charles and Bhagwat, 1994).

Dynamic Source Routing Protocol (DSR): DSR is similar to AODV except so it forms routes on demand when requested is generated. Rather than using routing tables at each intermediate node, it uses source routing. Source routing determination requires accumulating the address between the origin and the destination of every node .The nodes processing the route discovery packets cache the accumulated path information. Thus to route packets, the learned paths are used. Since all routing information is maintained at mobile nodes, this protocol is dependent on source routing.

The comparison of the three routing protocols DSDV, DSR and AODV is shown in Table 1.

Ad-Hoc on Demand Distance Vector Routing Protocol (AODV): AODV uses an on demand approach for finding routes. It's capable of both unicast and multicast routing. To identify the most recent path, it employs destination sequence number. In AODV for data packet transmission, corresponding to each flow the origin node and the destination nodes store the next-hop information.

From just one Route-Request it may obtain multiple routes to different destinations.it uses destination sequence number to determine up-to-date way to the destination. If the destination sequence quantity of the present packets received is greater compared to last destination sequence number stored at the node only then the path information is updated by a node.

Table1: Property comparison of DSDV, DSR and AODV

[4] [6]

\begin{tabular}{|l|l|l|l|}
\hline \multicolumn{1}{|c|}{$\begin{array}{c}\text { Protocol } \\
\text { property }\end{array}$} & \multicolumn{1}{|c|}{ DSDV } & \multicolumn{1}{|c|}{ DSR } & YDV \\
\hline Loop free & Yes & Yes & Yes \\
\hline $\begin{array}{l}\text { Multicast } \\
\text { routes }\end{array}$ & No & Yes & No \\
\hline Distributed & Yes & Yes & Yes \\
\hline $\begin{array}{l}\text { Unidirectional } \\
\text { link support }\end{array}$ & No & Yes & No \\
\hline Multicast & No & No & Yes \\
\hline $\begin{array}{l}\text { Periodic } \\
\text { broadcast }\end{array}$ & Yes & No & Yes \\
\hline QoS support & No & No & No \\
\hline $\begin{array}{l}\text { Routes } \\
\text { maintained in }\end{array}$ & Route table & Route cache & Route table \\
\hline
\end{tabular}




\begin{tabular}{|l|l|l|l|}
\hline $\begin{array}{l}\text { Route cache } \\
\text { timer }\end{array}$ & Yes & No & Yes \\
\hline Reactive & No & Yes & Yes \\
\hline
\end{tabular}

\section{LITERATURE REVIEW}

The aim of the literature review is to locate and explore the QoS in Wireless Mesh Networks and also what're the various problems in existing protocols and techniques. The key goal with this literature review is to get the gaps in existing research and methods and also what would be the possible methods to overcome these holes.

Nivedita Gaur et al. (2014) [1] proposed a Load-aware NonPersistent small-world long- link Routing (LNPR) algorithm for small-world wireless mesh networks to reach lower average transmission path length for data transfer sessions among some source-node and destination- node pairs in the network. LNPR uses load balancing strategy to raised distribute the network traffic on the list of normal-links and the non-persistent long-links in the small-world wireless mesh networks for efficient use of long-links which are precious data transmission paths in the network. LNPR provides $58 \%$ to $95 \%$ improvement in call blocking probability and $23 \%$ to $70 \%$ in maximum load reduction with increment which range from only $0.7 \%$ to $9 \%$ increase in average transmission path length. Small-world wireless mesh networks find numerous applications in rural and community networks for costeffective communication.

B.Nandini et al. (2014) [2] presented common metrics considered in routing protocol design and complete comparative analysis between some of the most deployed routing protocols in the wireless mesh networks along with simulation results for throughput, overhead and overall delay.

Lekha V. Bhandari et al. (2013) [3] proposed various protocol challenges and the comparative studies of numerous Protocols such as Simple Opportunistic Adaptive Routing protocol (SOAR), MAC, MPR, GPR etc on the foundation of numerous parameters.

P.K. Bedi et al. (2012) [4] have studied the different issues linked to routing protocols, existing routing protocols and routing metrics for WMN. Their studied end up being of great aid in deciding which routing protocol and routing metric will be useful where situation. This paper also serve as a baseline for developing a routing protocol that takes care of all the problems linked to routing.

Govindaraj. E. et al.(2012) [5] have proposed a QoS aware robust multipath routing algorithm for wireless mesh networks. The goal of the protocol is to offer a QoS constrained route from source to the destination. So a multiple disjoint paths for a source destination pair. Hence for initial time interval, probe packets are sent along all paths simultaneously from the origin to the destination. On receiving the probe packets, cumulative transmission energy, average delay and bandwidth are estimated for every path and a feedback report is provided for the destination. Therefore from the multiple paths, the robust best path is selected on the basis of the feedback report from the destination. Additionally it detects the changes in the path quality that hinders QoS requirements of the flows and reroutes the flow through alternative robust routes.

S.K.Gupta et al. (2011) [6] simulated and analyzed DSDV and AODV routing protocol using different parameter of QoS metrics. As a reactive protocol AODV transmited network information only on-demand and DSDV maintained table driven routing mechanism as proactive routing protocol. Two kinds of data packet TCP and UDP have now been analyzed.. DSDV and AODV routing protocol, packet delivery ratio is not dependent of offered traffic load. AODV protocols deliver $70 \%$ to $90 \%$ of the packets in all cases, while DSDV delivering $50 \%$ to $75 \%$. Delay is high initially in AODV but over time it is very low. But in case of DSDV, it is very low at starting and increased gradually specially for UDP packets. DSDV gave better jitter performance as a result of low node mobility and free channel, but dissimilarity of the packets arrival time or jitter was tiny bit high in case of AODV because of high node mobility and unavailability of free channel. So that they figured AODV indicating its highest efficiency and performance under high mobility than DSDV. Simulation results showed the performance of TCP and UDP packets with respect to the common end-to-end delay, throughput and jitter.

I.Cooper et al. (2011) [7] considered the optimisation of transmission schedules for infrastructure Wireless Mesh Networks by which data is forwarded through mesh routers from an individual Internet Gateway node. The mesh routers receive and aggregate data from local mobile phones and each mesh router has an assigned data allowance to make certain fairness, set according to its geographical position or the predicted usage patterns. They examined the utilization of fair and efficient link scheduling for Wireless Mesh Networks and offer an integer program for maximising the throughput allowance for each mesh router in a network given the topology. This system runs on the slotted time approach to maximise the throughput within certain number of slots $\mathrm{N}$, thus allowing a network to be split into sub networks for local use of the mesh routers, and back-haul transmissions to the gateway. Answers are presented showing the optimised throughput for a choice of networks and a selection of values for $\mathrm{N}$.

GhoshSaurav et al.(2011) [8] have studied wireless mesh networks as a powerful methods to provide broadband internet. Connectivity, reliability and throughput being the major QoS concern areas in WMNs. WMNs use orthogonal communication channels and multiple radios to boost throughput, reduce interference and provide path redundancy, connectivity and reliability. A cluster-based approach is employed to divide the network into clusters hence localising the channel assignment problem in each cluster.

S.Kannan et al.(2011) [9] have discussed that Mobile Ad-hoc Networks (MANET) as self-organizing and self-configuring multi-hop wireless networks. Mainly due to the mobility of the nodes, the structure of the network changes dynamically. In mobile ad-hoc networks, a routing procedure is always needed to locate a path so as to forward the packets appropriately between the origin and the destination. Route changes and temporary link failures occur frequently in a MANET. Assuming that most packet losses are as a result of congestion, in this environment TCP performs poorly. So a fresh mechanism TASR, TCP-aware source routing is proposed that could improve TCP performance in wireless Ad-hoc networks. So to reduce out-of-ordered packets, consecutive timeouts and retransmissions in TCP, TASR adds a hold state to a preexisting routing protocol. It includes route discovery and route maintenance.

S.Kannan et al.(2010) [10] have studied Mobile Ad-hoc Network routing protocol. The main objective of the protocol is always to overcome the hurdles created by the dynamically changing topology and hence create an efficient and correct 
communication path between any two nodes keeping because minimum routing overhead and bandwidth consumption. Since an ad-hoc environment offers various challenges that do not exists in fixed networks, the style problem so the routing protocol is not so simple. So several routing protocols have now been proposed with this purpose. The deep study and the comparison of the performance of the protocols have now been done. Various protocols are Destination Sequenced Distance Vector, Ad-Hoc Demand Distance Vector, Dynamic Source Routing, etc.

IshitaBhakta et al.(2010) [11] have studied Wireless mesh networks (WMNs) that includes mesh clients that may be either stationary or mobile, static mesh routers through which the clients communicate with one another and with the other networks or Internet. Multi-hop communication, the existence of inherently error prone wireless channel, the static nature of mesh nodes, the accessibility to sufficient bandwidth resources and the need to transport multimedia traffic with different QoS budgets necessitate the style of QoS-aware routing metrics to improve the performance of routing algorithms. The detailed study of the motivations for new and quality-aware routing metrics has been done and provides with a detailed comparative analysis of some routing metrics that attempt to address these goals.

S.Kannan et al.(2010) [12] have studied and compared the performance of numerous mobile ad-hoc network routing protocols like AODV, DSI and DSDV. Meeting the challenges of the changing topology, the routing protocol aims at providing a correct and efficient communication path between any two existing nodes of the network hence minimising bandwidth consumption and routing overhead.

S.Karthik et al.(2010) [13] have discussed mobile ad-hoc network as a network that does not require any infrastructure or central administration, hence they are suitable limited to providing temporary communication links. The main problem area in computer network is to design the network in this way so it can efficiently cope up with the speed issue that's mainly required today. In a network the data packets have to be routed to the destination with minimum loss and delay and maximum packet delivery ratio, so there must exist an efficient robust and adaptive routing algorithm that satisfies all the quality of service requirements. Inspired by the behaviour of biological ants, Multi Agent Ant Based Routing Algorithm was created from the ACO framework that contains both proactive and reactive components. This technique increases packet delivery ratio, node connectivity and decreases average end to get rid of delay. As node connectivity increases, the packet loss is reduced.

RonghuiHou eta al.(2009) [14] has described hop-by-hop bandwidth guaranteed routing protocol in IEEE 802.11-based wireless mesh networks. Bandwidth is neither concave nor additive in wireless networks as a result of interference among links. Bandwidth must be isotonic, the required and sufficient property for consistent hop-by-hop routing which will be unfortunately not available. So to fix the situation an isotonic parameter is introduced that captures the available bandwidth metric in order that packets can traverse the most and width path consistently in line with the routing tables constructed in the nodes over the path.

Chi Harold Liu et al.(2009) [15] have studied Cross-layer design for quality of service (QoS) in wireless mesh networks which can be expected to guide various forms of applications with different and multiple QoS and grade-of-service (GoS) requirements. In order to achieve the exact same various technologies have now been exploited and algorithm must be designed. Since a lot of the existing works on cross-layer design focus on the interaction of up to two layersWhile the GoS concept is overlooked in wireless mesh networks, so a unified framework is proposed that exploits the physical channel properties and multi-user diversity gain of WMNs and by performing intelligent route selection and connection admission control provides both QoS and GoS to many different applications.

Chi Harold Liu et al.(2008) [16] have discussed Wireless Mesh Networks that support various forms of applications with different quality of service requirements. The inefficiency of the layered approaches that overlook the interaction between the routing algorithms and medium access control (MAC) has generated the look of cross-layered approaches. So a distributed, multi-constrain, cross-layer QoS routing algorithm is proposed that could simultaneous satisfy multiple QoS requirements. Thus efficiently proving various QoS requirements and achieving higher network throughput.

AnandPrabhuSubramaniam et al. (2008) [17] have studied multi-hop WMNs where each node is built with multiple radio interfaces and multiple channels readily available for communication. So there exists the situation of assigning channels to the communication links in the network. The number of radios on any node could be less than how many available channels, obeying the constraint that how many different channels assigned to the link is practically how many radio interfaces of the node. So for the channel assignment, centralised and distributed algorithms are designed.

U.Ashraf et al. (2008) [18] proposed a fresh route stability mechanism which gives significant improvements in existing mesh routing protocols by making routes resilient to transient transmission failures which occur due to congestion. Simulation results showed a marked improvement of up to 75 percent in throughput and around 40 percent reduced delays.

Wai-Hong Tam et al.(2007) [19] have studied the wireless mesh network that have throughput increase as an open and challenging research issue. The optimal solution being is to enable transceivers to efficiently utilize multiple channels dynamically. As the existing work does not think about the routing issue, so from the part of end-to end throughput the benefits of multipath routing in multi-channel WMNs are exploited proposing Joint Multi-channel and Multi-path control (JMM) protocol that combines multi-channel link layer with multi-path routing.

Parag S. Mogre et al.(2007) [20] have studied the wireless mesh networks, the many challenges that exists to supply QoS. So while developing any algorithm for supporting Qos, care needs to be studied that it's in respect to the standards and has appropriate mechanism for the development of WMNs. So the research challenges highlight pitfalls and give pointers to appreciate QoS in WMNs.

VinodKone et al.(2007) [21] have studied WMNs that could provide seamless broadband connectivity to the network users with low maintenance and setup cost. To aid the actual time requirements the WMNs will need to have improved quality of service guarantees. So a routing protocol QUORUM is proposed that provides accurate QoS properties by predicting correctly the delay and loss characteristics of data traffic. It integrates a novel end-to-end packet delay estimation mechanism with stability aware routing policies which allows to more accurately followQos requirements by minimising misbehaviour of selfish nodes. 
Krishna N. Ramachandran et al.(2006) [22] have studied the capacity problem in WMNs, that may be reduced by equipping the mesh routers with multiple radios tuned to nonoverlapping channels. The channel assignment presents difficult as co-located wireless networks are apt to be tuned to the same channels. The performance is adversely affected by the escalation in interference. So an interferenceaware channel assignment algorithm and protocol is presented for multi-radio WMNs that address the interference problem. It assigns channels to the radios to minimise interference within the mesh network and involving the mesh network and co-located wireless networks. It uses a novel interference estimation technique implemented at each mesh router.

Jian tang et al.(2005) [23] have studied the throughput in WMNs that may be improved by multi-channel communications when compared with single-channel communications. The influence of the interference can be reduced by the use of multiple channels. The interference aware topology control and QoS routing in IEEE 802.11 based multi-channel wireless mesh networks with dynamic traffic has been studied. In such networks, channel assignment and routing are the 2 basic issues to be dealt with. Different channel assignment can result in different network topologies. So a novel definition of the co-channel interference is presented.

\section{GAPS IN EXISTING LITERATURE}

The survey has shown that the most of QoS aware routing protocols has certain limitations. Following are the main limitations in earlier work:

1. Congestion Control: In case the congestion is the biggest issue that deteriorates the quality of service. The packet delay, loss and blocking of new connections are the typical effects.

2. Secure Transmission: The data send over the network needs to be confidential, so a secure channel or a confidential channel is required for data transmission.

3. Collision Avoidance: To avoid resource contention such that to avoid simultaneous attempts to access the same resource.

4. Flooding: There is graceful performance degradation during overload. So for better throughput and better performance the system needs to be modified to work in the hazardous conditions like overflow.

\section{PROBLEM DEFINITION}

Wireless Mesh Network (WMN) is a key technology, supporting a variety of several emerging and commercially interesting applications e.g., neighbourhood networks, community and broadband home networking. It has emerged as a flexible, reliable and cost effective way of providing broadband internet access over wide areas through multi hop communication. To provide adaptive and flexible wireless Internet connectivity, it has been evolved as one of the most promising concepts for auto-configurable and self-organizing wireless network with multi hop and mesh technology. Network connectivity is automatically maintained and established by all the nodes in the mesh network. It also serves as a cost effective technique for establishing robust and reliable broadband access between Internet service providers (ISPs) and other end-users.

The main objectives of QoS based routing are optimal utilization of resources for improving total network throughput and graceful performance degradation during overloading conditions offering better throughput, Dynamic determination of feasible paths for accommodating the QoS of the given flow under various policy constraints such as provider selection, path cost etc. The overall objective of this dissertation is to propose a new multi-radio, multi-channel and clustering based wireless mesh protocol to improve the QoS further. This dissertation has considered the DSDV protocol to locate the secure and optimised path. The proposed technique also utilizes the LZW based lossless data compression and intra cluster data aggregation to enhance the communication between the source and the destination. The use of clustering has the ability to aggregates the multiple packets and locate a single route using the clusters to improves the intra cluster data aggregation. The utilization of the LZW based loss less data compression has ability to reduce the data packet size so will consume lesser energy thus increase the network QoS.

\section{CONCLUSION AND FUTURE WORK}

This paper has reviewedQoS issue of WMNs. WMNs have emerged as a flexible, reliable and cost effective way of providing broadband internet access over wide areas through multi hop communication. This paper has reviewed different routing protocols used in the WMNs. The review has shown that the existing work has neglected many issues. No technique is effective of every kind of circumstances. Congestion, secure transmission, collision avoidance and flooding have been found a critical issue to gain QoS.

In near future we will modify the existing routing techniques using cluster based data aggregation to enhance the QoS of WMNs further.

\section{REFERENCES}

[1] Nivedita Gaur, Abhishek Chakraborty, and B. S. Manoj, "Load-aware Routing for Non-Persistent Small-World Wireless Mesh Networks", In International Electrical and Electronics Engineers (IEEE), 2014.

[2] Nandini, Prof. Suresh Pabboju, and Dr.G.Narasimha, "Routing protocols for Wireless Mesh Networks: A comparative study", In International Journal of Multidisciplinary and Current Research, Vol.2, 2014.

[3] Lekha V. Bhandari and Avinash P. Wadhe, "Protocol Analysis in Wireless Mesh Network", In International Journal of Application or Innovation in Engineering \& Management, Vol.2, Issue4, 2013.

[4] P.K. Bedi, Yadu Nagar, Amit and Rajni Yadav, "Study of Routing Protocols: Single and Multipath for WMN",In International Journal of Computer Science \& Applications, Volume1, 2012.

[5] E., Govindaraj. , V. P. Arunachalam and S. Karthik. "A QoS Aware Robust Multipath Routing Protocol for Wireless Mesh Networks." European Journal of Scientific Research Vol.78 No.2 (2012), pp.222-231.

[6] Gupta, Sachin Kumar, and R. K. Saket, "Performance metric comparison of AODV and DSDV routing protocols in manets using ns-2", International Journal of Research and Reviews in Applied Sciences, Vol.7, Issue3, pp: 339-350, 2011.

[7] Ian Cooper, Stuart Allen, and Roger Whitaker, "Optimised Scheduling for Wireless Mesh Networks using Fixed Cycle Times", In Institute of Electrical and Electronics Engineers, 2011. 
[8] Saurav, Ghosh, Das Niva, and SarkarTanmoy. "A Cluster Based Multi-Radio Multi-Channel Assignment Approach in Wireless Mesh Networks." In Computer Networks and Information Technologies, pp. 487-491. Springer Berlin Heidelberg, 2011.

[9] Kannan, S., S. Karthik, and V. P. Arunachalam. "An Enhanced Packet Retransmission Method for Improving TCP-Aware Source Routing in Mobile Ad-Hoc Network." Asian Journal of Information Technology 10, no. 1 (2011): 20-25.

[10] Kannan, S., S. Karthik, and V. P. Arunachalam. "Performance Analysis and Comparison of Mobile Ad hoc Network Routing Protocols." Research General of Applied Sciences 6, no. 5 (2010): 424-428.

[11] Bhakta, Ishita, KoushikMajumdar, A. Kumar BHATTACHARJEE, Arik Das, D. KUMAR SANYAL, MatanginiChattopadhyay, and SamiranChattopadhyay. "Incorporating QoS Awareness in Routing Metrics for Wireless Mesh Networks." In Proceedings of the World Congress on Engineering, vol. 1. 2010.

[12] Kannan, S., S. Karthik, and V. P. Arunachalam. "An Investigation on Performance Analysis and Comparison of Proactive and Reactive Routing Protocols in Mobile Ad-Hoc Network." International Journal of Soft Computing 5, no. 5 (2010): 194-199.

[13] Kannan, S., T. Kalaikumaran, S. Karthik, and V. P. Arunachalam. "Ant colony optimization for routing in mobile ad-hoc networks." International Journal of Soft Computing 5, no. 6 (2010): 223-228.

[14] Hou, Ronghui, King-Shan Lui, Hon-Sun Chiu, Kwan L. Yeung, and Fred Baker. "Routing in multi-hop wireless mesh networks with bandwidth guarantees." In Proceedings of the tenth ACM international symposium on Mobile ad hoc networking and computing, pp. 353354. ACM, 2009.

[15] Liu, Chi Harold, AthanasiosGkelias, Yun Hou, and Kin $\mathrm{K}$. Leung. "Cross-layer design for QoS in wireless meshes networks." Wireless personal communications 51, no. 3 (2009): 593-613
[16] Liu, Chi Harold, Kin K. Leung, and AthanasiosGkelias. "A novel cross-layer QoS routing algorithm for wireless meshes networks." In Information Networking, 2008. ICOIN 2008. International Conference on, pp. 1-5. IEEE, 2008.

[17] Subramanian, AnandPrabhu, Himanshu Gupta, Samir R. Das, and Jing Cao. "Minimum interference channel assignment in multiradio wireless mesh networks." Mobile Computing, IEEE Transactions on 7, no. 12 (2008): 1459-1473.

[18] Usman Ashraf, Slim Abdellatif and Guy Juanole , "Route Stability in Wireless Mesh Access Network", In International Conference on Embedded and Ubiquitous Computing, 2008

[19] Tam, Wai-Hong, and Yu-Chee Tseng. "Joint MultiChannel Link Layer and Multi-Path Routing Design for Wireless Mesh Networks." In INFOCOM, pp. 20812089. 2007.

[20] Mogre, Parag S., Matthias Hollick, and Ralf Steinmetz. "QoS in wireless mesh networks: challenges, pitfalls, and roadmap to its realization." In 17th International Workshop on Network and Operating Systems Support for Digital Audio \& Video,Urbana-Champaign, Illinois, USA. 2007.

[21] Kone, Vinod, Sudipto Das, Ben Y. Zhao, and HaitaoZheng. "QUORUM: quality of service in wireless mesh networks." Mobile Networks and Applications 12, no. 5 (2007): 358-369.

[22] Ramachandran, Krishna N., Elizabeth M. Belding-Royer, Kevin C. Almeroth, and Milind M. Buddhikot. "Interference-Aware Channel Assignment in Multi-Radio Wireless Mesh Networks." In INFOCOM, vol. 6, pp. 112. 2006.

[23] Tang, Jian, GuoliangXue, and Weiyi Zhang. "Interference-aware topology control and QoS routing in multi-channel wireless mesh networks." In Proceedings of the 6th ACM international symposium on Mobile ad hoc networking and computing, pp. 68-77. ACM, 2005. 